\title{
The Proposed Improvement to Achieve Maturity from Low Impact Program Management Office - Case: PT Angkasa Pura II
}

\author{
Diah Dwi Hapsari ${ }^{1}$, Aries F. Firman ${ }^{2}$ \\ ${ }^{1,2}$ School of Business Management, Institute Technology Bandung, Indonesia
}

\begin{abstract}
Many Big companies realize the importance of PMO. The word "P" in the PMO can stands as Project, Program or Portfolio, it depends on a company needs. project is establishment of structured process in order to generate particular outputs, program is compilation of several projects while portfolio is compilation of several programs (Tjahjana et al., 2009a). For Angkasa Pura II PMO stands for Program Management, the centralized program management office to take on responsibilities for program related functions and program-related activities.
\end{abstract}

KEYWORDS: Maturity Components, Project Management, Program Management, Program Management Office, PMO Maturity Level.

\section{INTRODUCTION}

PT Angkasa Pura II (APII) is one of Indonesia Airport company under ministry of State own company which manage 20 airports in the west side of Indonesia. The Establishment of PMO is important for company with many programs, especially with complex programs that involve many stakeholders. PMO will act as project or program orchestrator to integrate, consult and solve the bottle necking condition. The objectives of PMO are to drive the change and improve company performance through program or project. The existing impact of PMO in Angkasa Pura II is consider very low caused by several condition in PMO operation components i.e. no formal necessity for project charter, tools variety in Project management plan, no guidance/procedures/standards in project reporting, no software and system for PM, no standard of quality management, no acknowledgement in people success, no communication planning, issues handled in ad-hoc manners and the condition where project issues and risk are not addressed in standard and regular basis.

To overcome this situation Angkasa Pura II need to implement Project Management maturity to transform PMO into better condition. The objectives of the paper are to define the baseline maturity level of APII and criteria needed by APII to achieve higher maturity level. The main literature of this research are Project Management book of Knowledge $6^{\text {th }}$ (PMI, 2017), Project Management Maturity Model (Crawford, 2015), High Impact PMO-How Agile PMO Deliver value in a complex world (Husser, 2017). Several journal also use to give wider picture of PMO as a bridging tool and the importance of a company to have strong knowledge in project management and interdisciplinary knowledge within organization" (Sandhu \& Wikström, 2018)

This research used qualitative methodology with in-vivo and descriptive coding in content analysis (Saldana, 2016). Fish bone or also commonly known as Ishikawa diagram (Bose, 2012), utilize to find the root of problem and the criteria needed by APII to achieve higher maturity level. The Researcher will also propose implementation roadmap to transform APII from Level two as baseline level to Level three 2 as the higher maturity level for AP II.

\section{LITERATURE REVIEW}

A. The Theory of Black Label Burger Bottom Bun (Husser, 2017)

Phillipe Husser explain about how relevant the existence of PMO with burger bottom bun. When we order burger with number one quality of meat, the fresher vegetable in town and the most selected seasoning, can you imagine what happen to those number one ingredient without the bun? There will be no burger. The second question how we eat the burger when we exclude the bottom bun? All other layers of ingredient will be fall out, scattered and mesh. PMO role or existence can be describe as the bottom bun, its hold all the projects or program together to ensure the goals achievement. PMO is the essential foundation of complex projects, program or portfolio, imagine how burger without the bottom bun the same as projects without PMO 


\section{International Journal of Current Science Research and Review}

ISSN: 2581-8341

Volume 05 Issue 01 January 2022

DOI: 10.47191/ijcsrr/V5-i1-32, Impact Factor: 5.825

IJCSRR@ 2022

www.ijesrr.org

\section{B. The PMO Challenge (Husser, 2017)}

Complexity of project always become the challenge for the PMO, the more complex projects/program in one organization the bigger challenge for the PMO. The complexity comes in various form such as below, the main challenge in project management originated from the feature complexity of the projects, programs, portfolio.

- The numbers of variable involve in the programs/projects i.e. the number of stakeholders either from internal or external company. From the internal there are the sponsor, the manager or leader, the PMO itself and project team members;

- irregular manners of interaction between variables, the relation of one variable to another variable may vary within one projects/program/portfolio to another and it became challenge for PMO to define the pattern.

- Unalterable prodigy, the challenge on complex project/program is when it already executes halfway and company realize it not suitable with company condition in need to be stop, we cannot go back to previous condition and this may cause chaotic situation

\section{PMO Responsibilities (Tjahjana et al., 2009b)}

PMO may varies between one company to other company it is of course customize following the condition of the company itself, how many projects or program they have and the degree of complexity of the project but the acrivities perform in the role of PMO generally are the same, even though the condition or structure for PMO in every organization is different one to another but in general the activities they are performing is the same includes coordinating resource, generate reports, coordination within stakeholders, monitoring and evaluation, giving recommendation to management and become the centre of Knowledge Management in the company.

\section{The Benefit of PMO (Tjahjana et al., 2009b)(Kwak \& Dai, 2000)}

The benefit of PMO is vary in a company, several effective PMO may achieve 70-80\% of stated benefit but most of them only get $50 \%$ even lower because many companies do not have clear understanding of the role of PMO itself while to make PMO work sufficient the company need to understand the role of PMO itself. To create a strong PMO the first thing a company needs to do is to have obvious understanding of PMO. PMO in an organization can act as central organizer, facilitate accurate communication among stakeholders, giving high level monitoring guarantee, and solve the bottle necking in project. PMO also have the benefit of providing consistent assistance on the projects.

\section{E. The Objectives of PMO (PMI, 2017)}

PMBOK has clearly state the transforming one company could achieve through program. A project or program should have transition company from current position to future better position. With the objectives of project and program as part of transformation the existence of PMO is to ensure the transition happened as targeted. The main purpose of PMO existence in company is to support organization's strategies in achieving its vision and mission, within company plan and PMO plan should be fit one another.

\section{F. PMO Maturity Level}

The evolution of Project Management in one organization typically lags behind any other capabilities within the company. A company only paid attention to PMO when there are critical condition that need PMO (Crawford, 2015, p. 3). This condition often make Project Management system in the company are not In place to support the need to practicing PM. With this condition the value of maturity assessment is needed. PMO Maturity level is a tool used to deal with the challenge faced by PMO.

When organization now their position for example a company in the level 2 of maturity, what is the most important things to know and what specific action should be taken to moved forward and be better. With PMO Maturity level organization has a vision and is moving to improve the capability of Project Management with exactly targeted efforts. Improving project management is like taking small step not giant leap. To reach the level 5 sometimes not needed because company will having more significant benefit from repeatable process level(Crawford, 2015).

Level-1 initial process: There are Recognition that project management process be found in the company, but no established practices and standard, individual project managers not held specific accountability by any process standards. 


\section{International Journal of Current Science Research and Review}

ISSN: 2581-8341

Volume 05 Issue 01 January 2022

DOI: 10.47191/ijesrr/V5-i1-32, Impact Factor: 5.825

IJCSRR@ 2022

www.ijcsrr.org

Level-2 Structured process and standards: Many projects management process found in the company, but not considered as organizational standards, documentation exist in basic process, management support the implementation of project management, but no consistent understanding, involvement \& organizational mandate to comply to all the projects.

Level-3 Organizational standards and institutional process: All Project Management process are in place and establish organizational standards, the process involved client and internal customers as active and intact members, almost all project uses the process with no exception. process and standards have been institutionalized by management. The process needs to be able to tailored to project characteristic, process cannot be applied equally to all projects considering different characteristic

Level-4 Manage Process: Project are managed with consideration as how they performed in the pass and what is expected in the future, management used efficiency and effectiveness metric to make decisions regarding projects and understand how the decision can affect others projects. Project information being integrated with other company information to give better information to management in making decision. Process is in place and being used actively to improve project management activities. To improved PM process, standards, and documentation,

Level-5: Lesson learned are regularly examined and used. Organization focuses not only on managing project effectively but also on continuous improvement.

\section{METHODOLOGY, DATA COLLECTION \& ANALYSIS}

\section{A. Methodology}

The research methodology is qualitative research where "the data collected particularly in the researcher's setting, data analysis develop inductively from specific general terms and the researcher create interpretation of the significance of the data" (Cresswell \& Cresswell, 2018, p. 43). Data use in this research include primary and secondary data.

For analysing the qualitative data author used coding method which combining the in vivo and descriptive coding in order to achieve the goals of the research as mention in the book by J. Saldana titled The Coding Manual for qualitative research "If needed, you can promote new or hybrid coding methods or adapt existing schemes, modified to suit the peculiar needs and disciplinary concerns of your research "(Saldana, 2016, p. 75)

In vivo coding use as the main method in this research where author take key words from what stated by interview respondent, "code in qualitative research is the majority a short words, or short phrase or suggestive attribute to one portion of language stand or visual sign" (Saldana, 2016, p. 4). Some keywords from in vivo coding being process to make sure it gets clearer meaning. The coding method only applied for primary data and literature review, secondary data use in this research only as strengthened factors for coding result.

\section{B. Data Collection}

Primary data collection in this research are get through depth interview method with selected respondent from internal Angkasa Pura II. Respondent are chosen based on strategic management process which are planning - implementation - evaluation

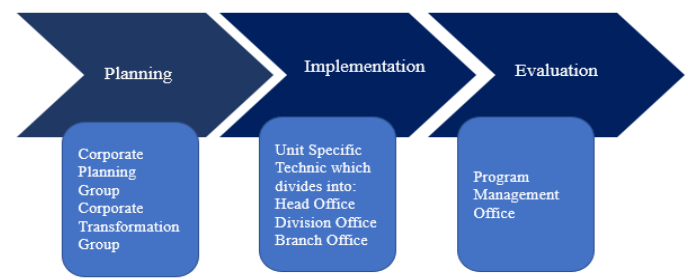

Figure 1. PMO Supply Chain Process

\section{Respondent profiling}

The respondent is chosen based on their interaction with PMO operation in order to get full view and completed information on how those people that represent function which close relation to PMO opinion and view on PMO. "the though behind qualitative research is purposely select participants or sites that will best help the researcher understand the problem and research question" (Cresswell \& Cresswell, 2018, p. 297). The respondent consists of nine employees of APII which divide into three group of respondents that are planning group, PMO group and User group. Respondent selection was carried out considering several matters: 


\section{International Journal of Current Science Research and Review}

ISSN: 2581-8341

Volume 05 Issue 01 January 2022

DOI: 10.47191/ijesrr/V5-i1-32, Impact Factor: 5.825

IJCSRR@ 2022

www.ijcsrr.org

the position in the company (VP, AVP, Program Controller), The age distribution (20-30, 30-40, and 40-50), Working experience (10-15 years, 16-20 years, 20-above years) and the structure (CEO Office, Division Office and Branch office). With these selection criteria author anticipates to get full view of PMO supply chain information.

\section{Expertise Interview}

Expert Interview is held to strengthening the output interview analysis it is the part of data analysis and triangulation process. The purpose of expertise interview is to strengthen the factors and criteria explain and mention by respondent and to get better analysis result. The reason on choosing CEO Angkasa Pura Sarana Digital to give expert opinion is based on his experience in research, business, projects and even academics.

\section{E. Interview Questions}

Interview question consist of six main question that aim to answer the research question and objectives at the end of data analysis. Consist of the criteria of high impact PMO, the most important criteria, PMO current position, things need to be improved, and PMO positioning in the company.

\section{F. Data Interpretation and analysis}

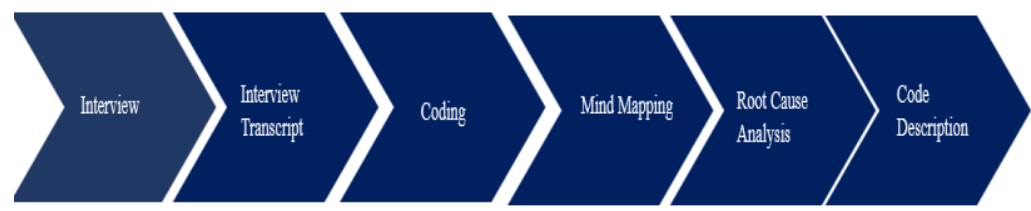

Figure 2. Data Interpretation \& analysis steps

Data interpretation and analysis steps:

1. Interview are put into recording to ensure all information are used and as interview evident. The interview recording then transfer into transcript;

2. Interview transcript are direct language of participants which being used(Saldana, 2016, p. 71) or word by word conveyed by respondent which transfer into the text. In transcript the statement of interview is used without any modification on the statement;

3. In-vivo and description coding are used to analyse the transcript. Code in qualitative research is most frequently a word or short phrase that symbolically stipulate a summative, prominent, essence-capturing, and/or powerful attribute for a portion of language based or visual data (Saldana, 2016, p. 4).

4. This research use hybrid coding method which in-vivo codes as the main coding method used for the interview transcript, the interview transcript author gets 43 codes. Descriptive coding used for field notes, documents, journal, books (Saldana, 2016, p. 72). The main data used in descriptive coding is PMBOK $6^{\text {th }}$ edition comprise the ten-knowledge area of project management as the framework: Project Integration management, Project scope management, project schedule Management, Project Quality Management, Project Resource Management, Project Communication Management, Project Risk Management, Project Procurement Management, Project Stakeholder Management.

5. Mind map is a diagram for reflecting, task words, concepts, or items related to and organized around a central construct or point using a non-linear graphical layout that allows the user to develop an intuitive framework around a central structure (mindmapping.com). The 43 codes from in-vivo coding put as sub-sub categories and used as central construction where descriptive codes sourced from PMBOK framework organized around the in-vivo coding to develop mind mapping.

\section{A. Preliminary PMO Maturity Assessment}

The self-assessment of PMO in APII goals is to have baseline level as starting point for APII to move forward to targeted level of maturity. The assessment delivered using components maturity in ten area of Project Management Knowledge and the result checked based on coding, mind mapping, root analysis, internal document and tacit knowledge. 
International Journal of Current Science Research and Review

ISSN: 2581-8341

Volume 05 Issue 01 January 2022

DOI: 10.47191/ijcsrr/V5-i1-32, Impact Factor: 5.825

IJCSRR@ 2022

www.ijcsrr.org

Table 1. Maturity self-assessment

\begin{tabular}{|c|c|c|c|c|c|}
\hline $\begin{array}{l}\text { Knowledge } \\
\text { Area }\end{array}$ & Components & Existing Condition & Reference & Coding & $\begin{array}{l}\text { Maturit } \\
\text { y Level }\end{array}$ \\
\hline & Main & & & & \\
\hline \multirow[t]{11}{*}{$\begin{array}{l}\text { Project } \\
\text { Integration } \\
\text { Management }\end{array}$} & $\begin{array}{l}\text { Project Charter } \\
\text { Development }\end{array}$ & $\begin{array}{l}\text { 1. There is no formal } \\
\text { necessity for project } \\
\text { charter } \\
\text { 2. Project Charter is } \\
\text { developed with no format } \\
\text { rules }\end{array}$ & $\begin{array}{l}\text { Interview } \\
\text { PMBOK }\end{array}$ & $\begin{array}{l}\text { Sub category } \\
-\end{array}$ & $\begin{array}{l}\text { Level } 1 \\
\text { Level } 1\end{array}$ \\
\hline & $\begin{array}{l}\text { Project } \\
\text { Management Plan } \\
\text { Development }\end{array}$ & $\begin{array}{l}\text { 1. Project management plan } \\
\text { varies } \\
\text { 2. Develop using WBS as } \\
\text { tools with basic milestone }\end{array}$ & $\begin{array}{l}\text { Interview } \\
\text { Interview }\end{array}$ & $\begin{array}{l}\text { Procedures } \\
\text { WBS }\end{array}$ & $\begin{array}{l}\text { Level } 1 \\
\text { Level } 1\end{array}$ \\
\hline & Project Execution & $\begin{array}{l}\text { Formal direction written in } \\
\text { RKAP, direction on BOD } \\
\text { meetings, formal letters }\end{array}$ & $\begin{array}{l}\text { SOP PMO, RKAP, } \\
\text { meeting minutes } \\
\text { Interview }\end{array}$ & $\begin{array}{l}\text { Project output } \\
\text { Project } \\
\text { outcomes } \\
\text { Performance } \\
\text { reports }\end{array}$ & Level 2 \\
\hline & $\begin{array}{l}\text { Monitoring \& } \\
\text { Controlling Project } \\
\text { Work }\end{array}$ & $\begin{array}{l}\text { Works result monitored } \\
\text { regularly in formal manners }\end{array}$ & $\begin{array}{l}\text { SOP Monitoring Day, } \\
\text { BOD-BOC Reports } \\
\text { Interview }\end{array}$ & Project output & Level 2 \\
\hline & $\begin{array}{l}\text { Integrated Change } \\
\text { Control }\end{array}$ & $\begin{array}{l}\text { Changes are communicated } \\
\text { formal way }\end{array}$ & $\begin{array}{l}\text { Internal letters } \\
\text { Interview }\end{array}$ & $\begin{array}{l}\text { Integration } \\
\text { Bottle necking } \\
\text { consultant } \\
\end{array}$ & Level 2 \\
\hline & $\begin{array}{l}\text { Project or Phase } \\
\text { Closure }\end{array}$ & $\begin{array}{l}\text { Final product delivery } \\
\text { formally }\end{array}$ & $\begin{array}{l}\text { Internal letters, PMO } \\
\text { email } \\
\text { Interview }\end{array}$ & $\begin{array}{l}\text { Performance } \\
\text { report }\end{array}$ & Level 2 \\
\hline & $\begin{array}{l}\text { Special: } \\
\text { (Project } \\
\text { Management } \\
\text { Office) } \\
\end{array}$ & $\begin{array}{l}\text { AP II formally has PMO in } \\
\text { their structure, defined } \\
\text { communicated throughout } \\
\text { organization }\end{array}$ & PMO Job Profile & & Level 3 \\
\hline & Project Support & $\begin{array}{l}\text { PMO give scheduling } \\
\text { assistant and developing scope }\end{array}$ & $\begin{array}{l}\text { SOP Monitoring \& } \\
\text { Evaluation, WBS } \\
\text { Form } \\
\text { Interview }\end{array}$ & Procedures & Level 3 \\
\hline & $\begin{array}{l}\text { Consulting \& } \quad \& \\
\text { Mentoring }\end{array}$ & $\begin{array}{l}\text { PMO is giving project } \\
\text { consulting in scheduling and } \\
\text { scope }\end{array}$ & $\begin{array}{l}\text { WBS Form } \\
\text { Interview }\end{array}$ & $\begin{array}{l}\text { Project } \\
\text { Consultant }\end{array}$ & Level 2 \\
\hline & $\begin{array}{l}\text { Process } \\
\text { Standards }\end{array}$ & $\begin{array}{l}\text { Basic PM standard, not } \\
\text { organizational } \quad \text { standards, } \\
\text { apply to all project monitored } \\
\text { by PMO }\end{array}$ & $\begin{array}{l}\text { SOP Monitoring \& } \\
\text { Evaluation } \\
\text { Interview }\end{array}$ & Procedures & Level 2 \\
\hline & Training & $\begin{array}{l}\text { Training for project managers } \\
\text { is carried out based on request }\end{array}$ & $\begin{array}{l}\text { Training schedule } \\
\text { Interview }\end{array}$ & $\begin{array}{l}\text { Project } \\
\text { Management }\end{array}$ & Level 2 \\
\hline
\end{tabular}


ISSN: 2581-8341

Volume 05 Issue 01 January 2022

DOI: 10.47191/ijcsrr/V5-i1-32, Impact Factor: 5.825

\begin{tabular}{|c|c|c|c|c|c|}
\hline & $\begin{array}{l}\text { Project } \\
\text { Management }\end{array}$ & $\begin{array}{l}\text { Guidance on project } \\
\text { management available to } \\
\text { individual based on request }\end{array}$ & Interview & $\begin{array}{l}\text { Project } \\
\text { Management } \\
\text { Procedures }\end{array}$ & Level 1 \\
\hline & $\begin{array}{l}\text { Project } \\
\text { Management } \\
\text { software tools }\end{array}$ & $\begin{array}{l}\text { There is no software in project } \\
\text { management, PM plan } \\
\text { develop manually using WBS } \\
\text { as tools }\end{array}$ & SOP WBS & & Level 1 \\
\hline \multirow[t]{6}{*}{$\begin{array}{l}\text { Scope } \\
\text { Management }\end{array}$} & $\begin{array}{l}\text { Scope } \\
\text { Management } \\
\text { Planning }\end{array}$ & $\begin{array}{l}\text { Scope management plan no } \\
\text { exist }\end{array}$ & $\begin{array}{l}\text { SOP WBS } \\
\text { Interview }\end{array}$ & $\begin{array}{l}\text { Project } \\
\text { Consultant } \\
\text { Work authority }\end{array}$ & Level 1 \\
\hline & $\begin{array}{l}\text { Requirements } \\
\text { Collection }\end{array}$ & $\begin{array}{l}\text { Undocumented business } \\
\text { collection }\end{array}$ & PMBOK & Internal Process & Level 1 \\
\hline & Scope Definition & Project scope part of WBS & $\begin{array}{l}\text { WBS document } \\
\text { Interview }\end{array}$ & $\begin{array}{l}\text { Project } \\
\text { Consultant }\end{array}$ & Level 1 \\
\hline & $\begin{array}{l}\text { Work Breakdown } \\
\text { Structure }\end{array}$ & Standard WBS exist & $\begin{array}{l}\text { WBS document } \\
\text { Interview }\end{array}$ & $\begin{array}{l}\text { Project } \\
\text { orchestrator } \\
\text { Project } \\
\text { consultant } \\
\text { Bottle necking }\end{array}$ & Level 2 \\
\hline & Scope Validation & $\begin{array}{l}\text { Method to validating project } \\
\text { are exist and consistently } \\
\text { applied to all project }\end{array}$ & $\begin{array}{l}\text { Project Evident, SOP } \\
\text { Monitoring \& } \quad \& \\
\text { Evaluation } \\
\text { Interview }\end{array}$ & $\begin{array}{l}\text { Monitoring } \\
\text { Evaluation } \\
\text { Performance } \\
\text { reports }\end{array}$ & Level 2 \\
\hline & $\begin{array}{l}\text { Scope } \quad \text { Change } \\
\text { Control }\end{array}$ & $\begin{array}{l}\text { Standard template exists to } \\
\text { monitored and evaluated } \\
\text { projects }\end{array}$ & $\begin{array}{l}\text { WBS document. } \\
\text { Reports } \\
\text { Interview }\end{array}$ & Authority & Level 2 \\
\hline \multirow[t]{5}{*}{$\begin{array}{l}\text { Time } \\
\text { Management }\end{array}$} & $\begin{array}{l}\text { Time Management } \\
\text { Planning }\end{array}$ & $\begin{array}{l}\text { Time management plan exist } \\
\text { as part of WBS }\end{array}$ & $\begin{array}{l}\text { WBS document } \\
\text { Interview }\end{array}$ & $\begin{array}{l}\text { Authority } \\
\text { Consultant } \\
\text { Orchestrator }\end{array}$ & Level 2 \\
\hline & Activity definition & $\begin{array}{l}\text { Activities define in WBS but } \\
\text { the defining method depends } \\
\text { on the experience and } \\
\text { knowledge of project manager }\end{array}$ & $\begin{array}{l}\text { WBS documents } \\
\text { SOP WBS } \\
\text { Interview }\end{array}$ & $\begin{array}{l}\text { Technical } \\
\text { competency } \\
\text { Project } \\
\text { management }\end{array}$ & Level 2 \\
\hline & $\begin{array}{l}\text { Activity } \\
\text { Sequencing }\end{array}$ & $\begin{array}{lrr}\text { Basic document } & \text { for } \\
\text { sequencing } & \text { activities } & \text { using } \\
\text { WBS } & & \end{array}$ & $\begin{array}{l}\text { WBS documents } \\
\text { Interview }\end{array}$ & $\begin{array}{l}\text { Managing } \\
\text { projects } \\
\text { Authority } \\
\text { Technical } \\
\text { competency }\end{array}$ & Level 2 \\
\hline & $\begin{array}{l}\text { Activity resource } \\
\text { estimation }\end{array}$ & $\begin{array}{l}\text { Activities are defined and but } \\
\text { resources define in ad-hoc } \\
\text { manners }\end{array}$ & $\begin{array}{l}\text { WBS document, SOP } \\
\text { WBS } \\
\text { Interview }\end{array}$ & $\begin{array}{l}\text { Technical } \\
\text { Consultant } \\
\text { Orchestrator } \\
\text { Performance }\end{array}$ & Level 1 \\
\hline & $\begin{array}{l}\text { Activity duration } \\
\text { estimation }\end{array}$ & No standard & Transcript & $\begin{array}{l}\text { Procedures } \\
\text { Policy } \\
\text { System }\end{array}$ & Level 1 \\
\hline
\end{tabular}


International Journal of Current Science Research and Review

ISSN: 2581-8341

Volume 05 Issue 01 January 2022

DOI: 10.47191/ijcsrr/V5-i1-32, Impact Factor: 5.825

IJCSRR@ 2022

www.ijesrr.org

\begin{tabular}{|c|c|c|c|c|c|}
\hline & $\begin{array}{l}\text { Schedule } \\
\text { development }\end{array}$ & $\begin{array}{lll}\text { Basic } & \text { guidelines } & \text { for } \\
\text { scheduling } & \text { using WBS } & \end{array}$ & $\begin{array}{l}\text { WBS document } \\
\text { Interview }\end{array}$ & $\begin{array}{l}\text { Managing } \\
\text { Authority } \\
\text { Consultant }\end{array}$ & Level 2 \\
\hline & Schedule Control & $\begin{array}{l}\text { Schedule are control using } \\
\text { WBS as guideline }\end{array}$ & $\begin{array}{l}\text { WBS document } \\
\text { Interview }\end{array}$ & $\begin{array}{l}\text { Authority } \\
\text { Structure }\end{array}$ & Level 2 \\
\hline & $\begin{array}{l}\text { Schedule } \\
\text { integration }\end{array}$ & $\begin{array}{l}\text { Schedule integration available } \\
\text { to several related projects only }\end{array}$ & $\begin{array}{l}\text { WBS document } \\
\text { Interview }\end{array}$ & $\begin{array}{l}\text { Integration } \\
\text { Authority }\end{array}$ & Level 2 \\
\hline \multirow[t]{4}{*}{$\begin{array}{l}\text { Cost } \\
\text { Management }\end{array}$} & $\begin{array}{l}\text { Cost Management } \\
\text { Planning }\end{array}$ & $\begin{array}{l}\text { Cost management on project } \\
\text { exist triggered by pandemic } \\
\text { covid-19 and an } \\
\text { organizational document }\end{array}$ & $\begin{array}{l}\text { Annual Budget Plan } \\
\text { document }\end{array}$ & & Level 2 \\
\hline & Cost estimating & $\begin{array}{l}\text { Some cost standards are } \\
\text { established }\end{array}$ & $\begin{array}{l}\text { Annual Budget Plan } \\
\text { documents }\end{array}$ & & Level 2 \\
\hline & $\begin{array}{l}\text { Budget } \\
\text { determination }\end{array}$ & $\begin{array}{lll}\text { Common practice } & \text { is } \\
\text { established } & & \\
\end{array}$ & $\begin{array}{l}\text { Annual } \\
\text { committee }\end{array}$ & & Level 2 \\
\hline & Cost control & No cost report & $\begin{array}{l}\text { Annual Budget Plan, } \\
\text { BOD } \\
\text { Minutes }\end{array}$ & & Level 2 \\
\hline \multirow{7}{*}{$\begin{array}{l}\text { Quality } \\
\text { Management }\end{array}$} & Main & & & & \\
\hline & $\begin{array}{l}\text { Quality } \\
\text { Management } \\
\text { Planning }\end{array}$ & $\begin{array}{l}\text { There is no standard of quality } \\
\text { management }\end{array}$ & $\begin{array}{l}\text { SOP } \\
\text { Interview }\end{array}$ & $\begin{array}{l}\text { Project } \\
\text { Management } \\
\text { Output } \\
\text { Outcomes } \\
\end{array}$ & Level 1 \\
\hline & Quality Assurance & $\begin{array}{l}\text { There are no practices } \\
\text { standard }\end{array}$ & Interview & $\begin{array}{l}\text { Procedures } \\
\text { Project } \\
\text { Management }\end{array}$ & Level 1 \\
\hline & Quality Control & No quality control in PM & $\begin{array}{l}\text { SOP Monitoring \& } \\
\text { Evaluation }\end{array}$ & & Level 1 \\
\hline & $\begin{array}{l}\text { Special interest: } \\
\text { Management } \\
\text { oversight }\end{array}$ & & & & \\
\hline & $\begin{array}{l}\text { Awareness and } \\
\text { support }\end{array}$ & $\begin{array}{l}\text { Basic tools provide by } \\
\text { management }\end{array}$ & $\begin{array}{l}\text { PMAO Application } \\
\text { Interview }\end{array}$ & $\begin{array}{l}\text { Technical } \\
\text { Leadership } \\
\text { Authority }\end{array}$ & Level 2 \\
\hline & involvement & $\begin{array}{l}\text { Management encourages } \\
\text { project performance reports }\end{array}$ & $\begin{array}{l}\text { PMO Reports } \\
\text { Interview }\end{array}$ & $\begin{array}{l}\text { Performance } \\
\text { Report } \\
\text { Leadership } \\
\text { Authority }\end{array}$ & Level 2 \\
\hline $\begin{array}{l}\text { Human } \\
\text { Resources } \\
\text { Management }\end{array}$ & $\begin{array}{l}\text { HR Management } \\
\text { Planning }\end{array}$ & $\begin{array}{l}\text { Defining number of people in } \\
\text { project in the budget plan }\end{array}$ & $\begin{array}{l}\text { Budget plan report } \\
\text { Interview }\end{array}$ & $\begin{array}{l}\text { Strong structure } \\
\text { Experience } \\
\text { Knowledge } \\
\text { management } \\
\text { Capabilities } \\
\text { Resource } \\
\text { allocation }\end{array}$ & Level 2 \\
\hline
\end{tabular}


International Journal of Current Science Research and Review

ISSN: 2581-8341

Volume 05 Issue 01 January 2022

DOI: 10.47191/ijcsrr/V5-i1-32, Impact Factor: 5.825

IJCSRR@ 2022

www.ijcsrr.org

\begin{tabular}{|c|c|c|c|c|c|}
\hline & $\begin{array}{ll}\text { Project } & \text { Team } \\
\text { Acquisition } & \end{array}$ & $\begin{array}{l}\text { Ad-hoc process to define } \\
\text { resource allocation }\end{array}$ & $\begin{array}{ll}\text { BOD } & \text { Meeting } \\
\text { minutes } & \\
\text { Interview } & \end{array}$ & $\begin{array}{l}\text { Strong structure } \\
\text { Experience } \\
\text { Knowledge } \\
\text { management } \\
\text { Capabilities } \\
\text { Resource } \\
\text { allocation }\end{array}$ & Level1 \\
\hline & $\begin{array}{l}\text { Project Team } \\
\text { Development }\end{array}$ & $\begin{array}{l}\text { Team development develop in } \\
\text { ad-hoc manners }\end{array}$ & $\begin{array}{ll}\text { Team } & \text { Decree } \\
\text { documents } & \\
\text { Interview } & \end{array}$ & $\begin{array}{l}\text { Strong structure } \\
\text { Experience } \\
\text { Knowledge } \\
\text { management } \\
\text { Capabilities } \\
\text { Resource } \\
\text { allocation }\end{array}$ & Level 1 \\
\hline & $\begin{array}{l}\text { Project Team } \\
\text { Management }\end{array}$ & $\begin{array}{l}\text { No team development } \\
\text { standards }\end{array}$ & Transcript & $\begin{array}{l}\text { Strong structure } \\
\text { Experience } \\
\text { Knowledge } \\
\text { management } \\
\text { Capabilities } \\
\text { Resource } \\
\text { allocation }\end{array}$ & Level 1 \\
\hline & $\begin{array}{l}\text { Special interest } \\
\text { components: } \\
\text { Professional } \\
\text { Development } \\
\text { Management }\end{array}$ & & & & \\
\hline & $\begin{array}{ll}\text { Individual } & \text { PM } \\
\text { Knowledge } & \end{array}$ & $\begin{array}{l}\text { Some individuals recognized } \\
\text { by knowing more than others }\end{array}$ & $\begin{array}{l}\text { Talent Management } \\
\text { system } \\
\text { Transcript }\end{array}$ & $\begin{array}{l}\text { Knowledge } \\
\text { Management } \\
\text { Competency } \\
\text { Lesson Learned }\end{array}$ & Level 1 \\
\hline & $\begin{array}{lr}\text { Individual } & \text { PM } \\
\text { Experience } & \& \\
\text { Competence } & \end{array}$ & $\begin{array}{l}\text { There is no acknowledge in } \\
\text { people success }\end{array}$ & Transcript & $\begin{array}{l}\text { Knowledge } \\
\text { Management } \\
\text { Competency } \\
\text { Lesson Learned }\end{array}$ & Level 1 \\
\hline & $\begin{array}{l}\text { Corporate } \\
\text { initiatives for }\end{array}$ & $\begin{array}{l}\text { No corporate recognition on } \\
\text { individual achievement }\end{array}$ & $\begin{array}{ll}\text { Awards } & \text { system } \\
\text { decree } & \\
\end{array}$ & & Level 1 \\
\hline & \multicolumn{5}{|l|}{ PM Development } \\
\hline \multirow[t]{2}{*}{$\begin{array}{l}\text { Communicati } \\
\text { on } \\
\text { Management }\end{array}$} & $\begin{array}{l}\text { Communication } \\
\text { management } \\
\text { planning }\end{array}$ & 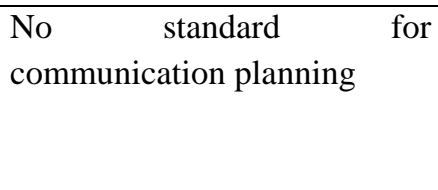 & transcript & $\begin{array}{l}\text { Communication } \\
\text { skill } \\
\text { Communication } \\
\text { channel }\end{array}$ & Level 1 \\
\hline & $\begin{array}{l}\text { Information } \\
\text { distribution }\end{array}$ & $\begin{array}{l}\text { Communication manages in } \\
\text { ad-hoc manners }\end{array}$ & transcript & $\begin{array}{l}\text { Communication } \\
\text { skill } \\
\text { Communication } \\
\text { channel }\end{array}$ & Level 1 \\
\hline
\end{tabular}


ISSN: 2581-8341

Volume 05 Issue 01 January 2022

DOI: 10.47191/ijcsrr/V5-i1-32, Impact Factor: 5.825

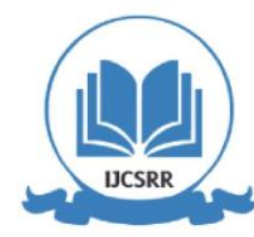

www.ijcsrr.org

\begin{tabular}{|c|c|c|c|c|c|}
\hline & $\begin{array}{l}\text { Communication } \\
\text { control }\end{array}$ & $\begin{array}{l}\text { Performance reporting include } \\
\text { issues only project status }\end{array}$ & $\begin{array}{l}\text { Reports } \\
\text { Transcript }\end{array}$ & $\begin{array}{l}\text { Communication } \\
\text { skill } \\
\text { Communication } \\
\text { channel }\end{array}$ & Level 1 \\
\hline & $\begin{array}{l}\text { Issues tracking } \\
\text { management }\end{array}$ & Issues handle on ad-hoc basis & $\begin{array}{lr}\text { BOD } & \text { Meeting } \\
\text { minutes, } & \text { internal } \\
\text { letters } & \\
\text { Transcript } & \end{array}$ & $\begin{array}{l}\text { Lesson Learned } \\
\text { Knowledge } \\
\text { Management }\end{array}$ & Level 1 \\
\hline \multirow[t]{6}{*}{$\begin{array}{l}\text { Risk } \\
\text { Management }\end{array}$} & $\begin{array}{l}\text { Risk Management } \\
\text { planning }\end{array}$ & $\begin{array}{l}\text { Development of RM part of } \\
\text { PM on large projects }\end{array}$ & $\begin{array}{l}\text { Risk reports } \\
\text { Transcript }\end{array}$ & $\begin{array}{l}\text { Evaluation } \\
\text { Performance } \\
\text { Output } \\
\text { Outcomes }\end{array}$ & Level 2 \\
\hline & Risk Identification & $\begin{array}{l}\text { Risk identification has been } \\
\text { documented for large projects }\end{array}$ & $\begin{array}{l}\text { Risk Reports } \\
\text { Transcript }\end{array}$ & $\begin{array}{l}\text { Evaluation } \\
\text { Performance } \\
\text { Output } \\
\text { Outcomes }\end{array}$ & Level 2 \\
\hline & $\begin{array}{l}\text { Qualitative } \quad \text { Risk } \\
\text { analysis }\end{array}$ & $\begin{array}{l}\text { Standard methodology on risk } \\
\text { analysis applied for large } \\
\text { projects }\end{array}$ & $\begin{array}{l}\text { Risk Reports } \\
\text { Transcript }\end{array}$ & $\begin{array}{l}\text { Evaluation } \\
\text { Performance } \\
\text { Output } \\
\text { Outcomes }\end{array}$ & Level 2 \\
\hline & $\begin{array}{l}\text { Quantitative Risk } \\
\text { analysis }\end{array}$ & $\begin{array}{l}\text { Standard methodology on risk } \\
\text { analysis applied for large } \\
\text { projects }\end{array}$ & Risk Report & & Level 2 \\
\hline & Risk Control & $\begin{array}{l}\text { Risk management plan } \\
\text { develop by assigned unit not } \\
\text { project team }\end{array}$ & $\begin{array}{l}\text { Job profile Risk Unit } \\
\text { Tacit } \\
\text { Transcript }\end{array}$ & $\begin{array}{l}\text { Evaluation } \\
\text { Performance } \\
\text { Output } \\
\text { Outcomes }\end{array}$ & Level 2 \\
\hline & $\begin{array}{l}\text { Risk } \\
\text { Documentation }\end{array}$ & $\begin{array}{l}\text { Historical data about risk } \\
\text { being documented }\end{array}$ & $\begin{array}{l}\text { Reports } \\
\text { Transcript }\end{array}$ & $\begin{array}{l}\text { Performance } \\
\text { Output }\end{array}$ & Level 2 \\
\hline \multirow[t]{4}{*}{$\begin{array}{l}\text { Procurement } \\
\text { and Vendor } \\
\text { Management }\end{array}$} & $\begin{array}{l}\text { Procurement } \\
\text { management } \\
\text { planning }\end{array}$ & $\begin{array}{l}\text { Procurement process are } \\
\text { determined but result is } \\
\text { control by project owner not } \\
\text { project manager }\end{array}$ & $\begin{array}{l}\text { BOD Decree } \\
\text { Transcript }\end{array}$ & $\begin{array}{l}\text { Communication } \\
\text { channel }\end{array}$ & Level 2 \\
\hline & $\begin{array}{l}\text { Procurement } \\
\text { requisition and } \\
\text { solicitation }\end{array}$ & $\begin{array}{l}\text { Scope approval and change } \\
\text { are issued part of TOR }\end{array}$ & $\begin{array}{l}\text { TOR } \\
\text { Transcript }\end{array}$ & $\begin{array}{l}\text { Communication } \\
\text { channel } \\
\text { Technical }\end{array}$ & Level 2 \\
\hline & $\begin{array}{l}\text { Procurement } \\
\text { control and vendor } \\
\text { management }\end{array}$ & $\begin{array}{l}\text { Vendors give regular reports } \\
\text { to program owner }\end{array}$ & $\begin{array}{l}\text { Progress reports } \\
\text { Transcript }\end{array}$ & $\begin{array}{l}\text { Communication } \\
\text { channel } \\
\text { Technical }\end{array}$ & Level 2 \\
\hline & $\begin{array}{l}\text { Procurement } \\
\text { Closure }\end{array}$ & Formal acceptance & $\begin{array}{l}\text { Acceptance letter } \\
\text { Transcript }\end{array}$ & $\begin{array}{l}\text { Communication } \\
\text { channel } \\
\text { Technical }\end{array}$ & Level 2 \\
\hline $\begin{array}{l}\text { Project } \\
\text { Stakeholder } \\
\text { Management }\end{array}$ & $\begin{array}{l}\text { Stakeholder } \\
\text { identification }\end{array}$ & $\begin{array}{l}\text { APII has standard in } \\
\text { stakeholder engagement in } \\
\text { general not specific for } \\
\text { particular project its cover all } \\
\text { stakeholders }\end{array}$ & $\begin{array}{l}\text { Stakeholder } \\
\text { engagement guidance } \\
\text { (SEG) } \\
\text { Transcript }\end{array}$ & $\begin{array}{l}\text { Communication } \\
\text { channel } \\
\text { Leadership }\end{array}$ & Level 2 \\
\hline
\end{tabular}




\section{International Journal of Current Science Research and Review}

ISSN: 2581-8341

Volume 05 Issue 01 January 2022

DOI: 10.47191/ijesrr/V5-i1-32, Impact Factor: 5.825

IJCSRR@ 2022

Www.ijcsrr.org

\begin{tabular}{|c|c|c|c|c|c|}
\hline & $\begin{array}{l}\text { Stakeholder } \\
\text { management } \\
\text { planning }\end{array}$ & $\begin{array}{l}\text { The process of communicate } \\
\text { with stakeholders varies but } \\
\text { the method is standardize }\end{array}$ & $\begin{array}{l}\text { SEG } \\
\text { Transcript }\end{array}$ & $\begin{array}{l}\text { Communication } \\
\text { channel } \\
\text { Leadership }\end{array}$ & Level 2 \\
\hline & $\begin{array}{l}\text { Managing } \\
\text { stakeholder } \\
\text { engagement }\end{array}$ & $\begin{array}{l}\text { Stakeholder relationship } \\
\text { strategies are in place but } \\
\text { varies }\end{array}$ & $\begin{array}{l}\text { SEG, SEP Reports } \\
\text { Transcript }\end{array}$ & $\begin{array}{l}\text { Communication } \\
\text { channel } \\
\text { Leadership }\end{array}$ & Level 2 \\
\hline
\end{tabular}

\section{B. Conclusion \& Summary}

\section{1) Conclusion}

Based on the discussion and analysis of the interview result, literature review, expertise opinion and secondary internal data, the author has concluded several points to answer research question:(1) The existing PM Maturity Level in Angkasa Pura II is level two. (2) The criteria needed by AP II to improve PM Maturity are: Strong role in project integration, enabling value creation, giving strategic recommendation to management, involvement in planning phase, become the source of organization knowledge management, strong communication skills, executing orchestrator and project consultant role in the company, become the bottle necking solution, creating good cross function coordination, the team mas must have strong technical knowledge in project management, strong leadership skills and possessed the culture of high learning.

\section{2) Recommendation}

Form overall analysis in this research there are four recommendations for APII: (1) To set target indicator based on the criteria as mention in conclusion. The criteria will be used as guidance for PMO to move forward and achieve level 3 PMO Maturity level (2) To follow up this research result with self-assessment of PMO Maturity Level with level three as the target (3) To accelerate achievement of level three maturity level, AP II should initiate PMO Champion Program as the part of Change Management Plan to ensure full participation and awareness of AP II employees (4) Develop implementation roadmap to achieve the maximum target of PM Maturity Level (level 5).

\section{REFERENCES}

1. Bose, T. K. (2012). Application of Fishbone Analysis for Evaluating Supply Chain and Business Process. https://doi.org/10.5121/ijmvsc.2012.3202

2. Crawford, J. K. (2015). Project Management Maturity Model, Third Eddition. In Taylor and Francis Group.

3. Cresswell, J. W., \& Cresswell, J. D. (2018). 5th edition Research Design Qualitative, Quantitative, and Mixed Methods Approaches (5th ed., Vol. 5). SAGE.

4. Husser, P. (2017). The High Impact PMO How Agile PMO Deliver Value in a complex world. CreateSpace Independent Publishing Platform.

5. Kwak, Y. H., \& Dai, C. X. Y. (2000). Assesing The Value of PMO.

6. Monteiro, A., Santos, V., \& Varajão, J. (2016). Project Management Office Models - A Review. Procedia Computer Science, 100, 1085-1094. https://doi.org/10.1016/j.procs.2016.09.254

7. PMI. (2017). Project Management Body of Knowledge (6th ed.). PM Institue, Inc.

8. Saldana, J. (2016). The Coding Manual for Qualitative researchers (J. Seaman (ed.); 3rd ed.). SAGE.

9. Sandhu, M. A., \& Wikström, K. (2018). Benchmarking the strategic roles of the project management office ( PMO ) when developing business ecosystems. https://doi.org/10.1108/BIJ-03-2018-0058

10. Tjahjana, L., Dwyer, P., \& Habib, M. (2009a). The Program Management Office Advantage. AMACOM.

11. Tjahjana, L., Dwyer, P., \& Habib, M. (2009b). The Program Management Office Advantage. AMACOM.

Cite this Article: Diah Dwi Hapsari, Aries F. Firman (2022). The Proposed Improvement to Achieve Maturity from Low Impact Program Management Office - Case: PT Angkasa Pura II. International Journal of Current Science Research and Review, 5(1), 275-284 